\title{
Une tragédie grecque
}

\section{Samia Hurst}

Prof. Dr méd., Institut Ethique Histoire Humanités (iEH2), Faculté de médecine, Genève

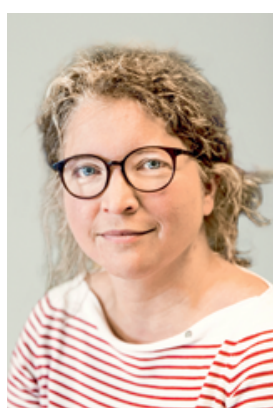

Aujourd'hui, je vais vous raconter une tragédie. Tout $\mathrm{du}$ moins une histoire où les personnes souffrent et sont le jouet de forces qui les dépassent. $\mathrm{XXI}^{\mathrm{e}}$ siècle oblige, ces forces ne seront pas des divinités capricieuses ni les lois du destin, mais celles du marché et de l'économie.

Premier acte: En décembre 2016, la filiale grecque de Novartis est accusée de corruption à large échelle dans le but de maintenir élevés les prix de certains médicaments oncologiques. Un des cadres convoqué par la justice fait une tentative de suicide. Il explique son geste en clamant qu'il ne veut pas être le bouc émissaire [1]. La maison mère de Novartis rappelle que l'entreprise est attachée «aux critères les plus élevés en matière de conduite éthique des affaires et de conformité réglementaire dans tous les aspects de son activité et prend très au sérieux toute allégation de mauvaise conduite». Elle annonce une pleine collaboration avec la justice.

Deuxième acte: Ce n'est en fait pas la première fois que Novartis est accusée de corruption. Sous enquête en Corée du Sud depuis l'été dernier, mise à l'amende en Chine en 2015, la firme a négocié une amende de 390 millions USD aux Etats-Unis après des accusations semblables. A chaque fois, la réaction est la même: il s'agit d'agissements locaux, face auxquels la tête de l'entreprise se distancie, rappelle que ses règles les interdisent [2], et promet une pleine collaboration avec la justice.

Lorsqu'une affaire de corruption survient dans une filiale locale, une part de la responsabilité morale repose donc bel et bien à la maison mère.

Troisième acte: Novartis n'est pas - de loin - la seule compagnie pharmaceutique a avoir été accusée ou mise à l'amende pour corruption récemment [3]. Selon un rapport publié l'an dernier par Transparency International [4], il semble que ce soit un problème de secteur. Que se passe-t-il, pour l'employé de l'industrie? Plus il est jugé au chiffre, plus il peut être tentant d'acheter du chiffre. S'il constate que ses concurrents le font en toute impunité, il peut se retrouver comme piégé. Sans doute lui dit-on effectivement que la corruption c'est mal. Si elle est profitable dans l'environnement réel où il travaille, en revanche, ce message là sera lui aussi reçu cinq sur cinq. Si l'entreprise n'est pas concrètement dissuasive, la distance qu'elle exprime quand ces faits se répètent deviendra de moins en moins crédible.

Lorsqu'une affaire de corruption survient dans une filiale locale, une part de la responsabilité morale repose donc bel et bien à la maison mère. Ici, elle est en Suisse. Dérangeant. Mais, me direz-vous: une entreprise entière, c'est aussi en concurrence avec d'autres. C'est aussi capable de constater que d'autres pratiquent la corruption et en profitent. Cela peut aussi, finalement, se retrouver piégé.

\section{La solution peut venir des états, mais elle pourrait aussi venir de l'industrie.}

Au premier acte, donc, nous pouvions être tentés d'accuser des personnes manquant de fibre morale. Au deuxième, de réprouver l'entreprise. Au troisième acte, nous devons pourtant constater que les responsables incluent les états, donc nous, et le marché, donc encore nous.

Encore plus dérangeant, donc. Que devrait-il se passer pour changer cette situation? La solution pourrait venir des états, mais elle pourrait aussi venir de l'industrie. Reconnaître légalement une responsabilité dans la prévention de ces cas, remplacer des amendes trop supportables par des sanctions mieux ciblées, comme des réductions de brevet: tout cela pourrait avoir un effet. Que pourraient faire les entreprises? Face à la concurrence, elles adoptent des comportements qui nuisent (à juste titre) à leur image et la raison principale est en fait que d'autres le font. Il est difficile de se retirer seul de ce petit jeu. Collectivement, par contre, cela devient réaliste. Une initiative crédible de coordination du secteur dans la lutte anti-corruption, ce serait une meilleure réponse la prochaine fois.

\section{Références}

1 https://www.rts.ch/info/economie/8282935-une-enquete-pourcorruption-presumee-visant-novartis-en-grece.html

2 https://www.novartis.com/about-us/corporate-responsibility/ doing-business-responsibly/ethics-compliance/anti-briberyanti-corruption

3 Dyer, O. Novartis is under investigation for allegedly bribing thousands of Greek doctors. BMJ 2017;356:j130.

4 http://www.transparency.org.uk/publications/corruption-inthe-pharmaceutical-sector/ 